\title{
Nonredundant roles of TIRAP and MyD88 in airway response to endotoxin, independent of TRIF, IL-1 and IL-18 pathways
}

\author{
Dieudonnée Togbe ${ }^{1}$, Gorse Aurore ${ }^{1}$, Nicolas Noulin ${ }^{1,2}$, Valérie FJ Quesniaux ${ }^{1}$, \\ Silvia Schnyder-Candrian ${ }^{1}$, Bruno Schnyder ${ }^{1}$, Virginie Vasseur ${ }^{1}$, Shizuo Akira ${ }^{3}$, \\ Kasper Hoebe ${ }^{4}$, Bruce Beutler ${ }^{4}$, Bernhard Ryffel ${ }^{1, *}$ and Isabelle Couillin ${ }^{1, *}$ \\ ${ }^{1}$ Molecular Immunology and Embryology, CNRS UMR6218, Transgenose Institute, Orleans, France; ${ }^{2} \& \& D$ \\ Department, Key-Obs, Orleans, France; ${ }^{3}$ Department of Host Defense, Research Institute for Microbial \\ Diseases, Osaka University, Suita, Osaka, Japan and ${ }^{4}$ Department of Immunology, Scripps Research Institute, \\ La Jolla, CA, USA
}

\begin{abstract}
Inhaled endotoxins induce an acute inflammatory response in the airways mediated through Toll-like receptor 4 (TLR4) and myeloid differentiation factor 88 (MyD88). However, the relative roles of the TLR4 adaptor proteins TIRAP and TRIF and of the MyD88-dependent IL-1 and IL-18 receptor pathways in this response are unclear. Here, we demonstrate that endotoxin-induced acute bronchoconstriction, vascular damage resulting in protein leak, Th1 cytokine and chemokine secretion and neutrophil recruitment in the airways are abrogated in mice deficient for either TIRAP or MyD88, but not in TRIF deficient mice. The contribution of other TLR-independent, MyD88-dependent signaling pathways was investigated in IL-1R1, IL-18R and caspase-1 (ICE)-deficient mice, which displayed normal airway responses to endotoxin. In conclusion, the TLR4-mediated, bronchoconstriction and acute inflammatory lung pathology to inhaled endotoxin critically depend on the expression of both adaptor proteins, TIRAP and MyD88, suggesting cooperative roles, while TRIF, IL-1R1, IL-18R signaling pathways are dispensable.
\end{abstract}

Laboratory Investigation (2006) 86, 1126-1135. doi:10.1038/labinvest.3700473; published online 18 September 2006

Keywords: endotoxin; innate immunity; airways response; TLR signaling; caspase-1

Inflammation of the airways due to environmental agents including endotoxin or lipopolysaccharide (LPS) plays an important role in the development and progression of chronic respiratory disease including asthma. ${ }^{1-5}$ Pathologic changes induced by endotoxin inhalation include acute respiratory distress syndrome, neutrophil recruitment, injury of the alveolar epithelium and endothelium with protein leak in the alveolar space. ${ }^{6,7}$ In mice, aerogenic exposure to endotoxin from Gram-negative bacteria induces acute pulmonary inflammation, local TNF production, alveolar-capillary leak and also a direct bronchoconstriction. ${ }^{8,9}$ Toll-like receptor (TLR) 4 and CD14 play a critical role in the pulmonary response to systemic endotoxin administration $^{10-12}$ and we showed recently that TLR4

Correspondence: Dr I Couillin, PhD, Molecular Immunology and Embryology, Transgenic Institute, CNRS UMR6218, 3 B Rue de la Ferollerie, Orleans Cedex 2 45071, France.

E-mails: couillin@cnrs-orleans.fr, bryffel@cnrs-orleans.fr

*Senior authorship shared IC and BR.

Received 25 May 2006; revised 13 July 2006; accepted 16 July 2006; published online 18 September 2006 expression level determines the extent of acute pulmonary response to inhaled endotoxin. ${ }^{13}$ Aerogenic endotoxin exposure also induces neutrophil recruitment into the alveolar space, ${ }^{8}$ which is MAPK-dependent, but independent of the secretion of TNF. ${ }^{9}$

TLR4 uses different combinations of TIR domain containing adaptor proteins to activate distinct signaling pathways, most prominently MyD88 and TIR domain containing adaptor inducing interferonbeta (TRIF), leading to the production of proinflammatory cytokines and type I IFNs, respectively ${ }^{14-17}$ Absence of MyD88 confers resistance to systemic endotoxin-induced shock. ${ }^{18}$ TIRAP-deficient mice are also resistant to the toxic effects of LPS, ${ }^{19}$ with defective induction of TNF, IL-6 or IL-12p40 and delayed activation of NF- $\kappa$ B and MAP kinases ${ }^{20,21}$ Indeed, MyD88 and TIRAP are involved in early activation of NF- $\kappa \mathrm{B}$ and MAP kinases ${ }^{19,21-23}$ whereas TRIF and TRAM are critical for late activation of $\mathrm{NF}-\kappa \mathrm{B}$ as well as IRF-3 activation. ${ }^{24,25}$

The inflammatory responses in lung, a site of continuous exposure to environmental antigens, are believed to be different from those present in less 
exposed, accessible sites. ${ }^{26,27}$ We showed recently that the TLR adaptor MyD88 is critical for the airway inflammatory response to endotoxins. ${ }^{28}$ MyD88 is at the crossroad of multiple TLR-dependent and TLRindependent signaling pathways, including IL-1R and IL-18R, or FAK. ${ }^{29}$ In certain infection models the extreme sensitivity of MyD88-deficient mice may be ascribed, at least in part, to deficient IL-1R/ IL-18R signaling pathways, as shown recently for cutaneous Staphylococcus aureus infection ${ }^{30}$ and our own unpublished results on mycobacterial infections (BR, VQ, DT, VV). Here, we investigated the underlying mechanisms of the MyD88-dependent airway inflammatory response. Using genetically modified mice we analyzed the relative contribution of the TLR4 adaptor proteins TIRAP, MyD88 and TRIF as well as the contribution of the MyD88-dependent, TLR-independent IL-1R and IL-18R signaling pathways on inhaled endotoxin induced lung injury.

TIRAP has recently been shown to be essential for LPS-induced lung inflammation. ${ }^{31}$ Here, we extend this observation to LPS induced bronchoconstriction and present a side-by-side evaluation of the several TLR4- and/or MyD88-dependent pathways potentially involved in these responses. We demonstrate that TIRAP together with MyD88 are both essential and nonredundant for LPS-TLR4-induced acute pulmonary inflammation response, while other signals contributing to TLR4- or MyD88dependent pathways such as TRIF, IL1-R and IL18R signals are dispensable. LPS-induced bronchoconstriction, pulmonary neutrophil sequestration, vascular leak, TNF, IL-12p40 and KC secretions were abrogated in either TIRAP- or MyD88-deficient mice while these parameters were unaffected in mice deficient for TRIF, IL1-R, IL-18R or ICE.

\section{Materials and methods}

\section{Mice}

TIRAP-/-, ${ }^{21}$ MyD88-/-, ${ }^{18}$ IL1-R1-/-, ${ }^{32}$ IL-18R-/-, ${ }^{33}$ caspase-1-/-, ${ }^{34}$ TLR4-/-, ${ }^{35}$ and Trif ${ }^{\text {Lps2 }}$ homozygote mutant mice ${ }^{15}$ backcrossed 10 times for MyD88-/- and TLR4-/-, and seven times for IL1-R1-/ - and caspase-1-/-, on the C57BL/6 genetic background and wild-type control C57BL/6 (WT) were bred in our animal facility at the Transgenose Institute (CNRS, Orleans). For experiments, adult (6-10 weeks old) mice were kept in sterile isolated ventilated cages. All animal experiments complied with the French Government's ethical and animal experiment regulations.

\section{Endotoxin Administration and Measurement of Airway Resistance}

Endotoxin $(10 \mu \mathrm{g})$ from Escherichia coli (serotype 055: B5, Sigma, St Louis, MO, USA) or saline were given by the aerogenic route using nasal instillation in a volume of $50 \mu \mathrm{l}$ under light i.v. ketamine-xylasine anesthesia. The airway resistance was evaluated by whole-body plethysmography ${ }^{9}$ at several time points. Unrestrained conscious mice were placed in wholebody plethysmography chambers (EMKA Technologies, France). Enhanced Respiratory Pause (PenH) measurements were collected over 3-6h. PenH can be conceptualized as the phase shift of the thoracic flow and the nasal flow curves. Increased phase shift correlates with increased respiratory system resistance. PenH is calculated by the formula $\mathrm{PenH}=$ $(\mathrm{Te} / \mathrm{RT}-1) \times \mathrm{PEF} / \mathrm{PIF}$, where Te is expiratory time, RT is relaxation time, PEF is peak expiratory flow, and PIF is peak inspiratory flow. ${ }^{36}$

\section{Bronchoalveolar Lavage Fluid}

Bronchoalveolar Lavage Fluid (BALF) was collected 3 or $24 \mathrm{~h}$ after endotoxin administration by canulating the trachea under deep i.p. ketamine-xylasine anesthesia and washing the lung four times with $0.5 \mathrm{ml}$ of saline at room temperature as described. ${ }^{36}$ The lavage fluid was centrifuged $10 \mathrm{~min}$ at 2000 r.p.m. $4{ }^{\circ} \mathrm{C}$ and the supernatant frozen for cytokine content. The cell pellet was resuspended in PBS, counted in a hematocytometer chamber and cytospin preparations were made using a Shandon cytocentrifuge (1000 r.p.m., $10 \mathrm{~min}$ ). The cells were stained with Diff-Quick (Dade Behring, Marburg, Germany). The supernatant was used for the measurement of cytokine and protein levels. TNF, IL-12 p40 and KC were measured by enzyme-linked immunosorbent assay (R\&D Duoset, Minneapolis, MO, USA). For protein determination, Bradford stain was added to the supernatant as described by the manufacturer (Bio-Rad, Ivry sur Seine, France) using an ovalbumin standard and absorbance was measured at $595 \mathrm{~nm}$ (Uvikon spectrophotometer, Kontron Zurich, Switzerland).

\section{Microscopy and Myeloperoxidase Activity in Lung}

After bronchoalveolar lavage and lung perfusion, the mice were sacrificed. The lung were fixed in $4 \%$ buffered formaldehyde for standard microscopic analysis, $3 \mu \mathrm{m}$ sections were stained with hematoxylin and eosin (H\&E) as described previously. ${ }^{36}$ Neutrophil and erythrocyte accumulation in alveoli, disruption of alveolar septae and activation of alveolar epithelial cells were quantified using a semiquantitative score with increasing severity of changes (0-5) by two independent observers including a trained pathologist (BR). Groups of five to eight mice per genotype and 10 randomly selected high-power fields $(\times 400$ magnification) per animal were analyzed.

Lung tissue MPO activity was evaluated as described..$^{12}$ In brief, the right heart ventricle was perfused with saline to flush the vascular content and lungs were frozen at $-20^{\circ} \mathrm{C}$ until use. Lung was 
homogenized by polytron, centrifuged and the supernatant was discarded. The pellets were resuspended in $1 \mathrm{ml}$ PBS containing $0.5 \%$ hexadecyltrimethyl ammonium bromide (HTAB) and $5 \mathrm{mM}$ ethylene-diamine tetra-acetic acid (EDTA). Following centrifugation, $50 \mu \mathrm{l}$ of supernatants were placed in test tubes with $200 \mu \mathrm{l}$ PBS-HTAB-EDTA, $2 \mathrm{ml}$ Hanks' balanced salt solution (HBSS), $100 \mu \mathrm{l}$ of $o$ dianisidine dihydrochloride $(1.25 \mathrm{mg} / \mathrm{ml})$, and $100 \mu \mathrm{l} \mathrm{H}_{2} \mathrm{O}_{2} \quad 0.05 \%$. After $15 \mathrm{~min}$ of incubation at $37^{\circ} \mathrm{C}$ in an agitator, the reaction was stopped with $100 \mu \mathrm{l} \mathrm{NaN} \mathrm{N}_{3} 1 \%$. The MPO activity was determined as absorbance at $460 \mathrm{~nm}$ against medium.

\section{BMDM Culture and Stimulation In Vitro}

Primary bone marrow-derived macrophages (BMDM) were obtained from femoral bone marrow as described. ${ }^{37}$ In brief, cells from the femur were isolated and cultured at $10^{6} \mathrm{cells} / \mathrm{ml}$ for 7 days in Dulbecco's minimal essential medium (DMEM, Sigma) supplemented with $20 \%$ horse serum and $30 \%$ L929 cell conditioned medium as a source of M-CSF. At 3 days after washing and reculturing in fresh medium, the cell preparation contained a homogenous population of $>95 \%$ macrophages. The BMDM were plated in 96-well microculture plates (at $10^{5}$ cells/well) and stimulated with LPS (E. coli, serotype 055 : B5, at $100 \mathrm{ng} / \mathrm{ml}$ ). Cell supernatants were harvested after 18-24 h of stimulation for TNF, IL-12p40 and KC measurement: analyzed immediately or stored at $-20^{\circ} \mathrm{C}$.

\section{Statistical Analysis}

Statistical evaluation of differences between the experimental groups was determined by MannWitney ' $U$ ' test for plethysmography experiments, and Student's $t$-test for others data, using Prism software. $P$-values of $<0.05$ were considered statistically significant.

\section{Results}

\section{Endotoxin-Induced Bronchoconstriction Depends on TIRAP and MyD88, not TRIF}

Intranasal administration of endotoxin (10 $\mu \mathrm{g}$ LPS) induced direct bronchoconstriction in C57BL/6 wildtype mice within $90 \mathrm{~min}$, whereas TIRAP-/- mice were completely unresponsive. LPS-induced bronchoconstriction increased for the next hours with a maximum enhanced pause (PenH) around $150 \mathrm{~min}$ and then decreased towards basal level after $4 \mathrm{~h}$ (Figure 1a). Bronchoconstriction was absent in TIRAP-/- mice (Figure 1a) and the difference to wild-type mice as represented by the area under the curve (AUC) was highly significant (Figure 1b). Similarly, MyD88-/- and TLR4-/- mice were also unresponsive to endotoxin, while Trif ${ }^{\text {Lps } 2}$ homozygote mutant mice functionally deficient for TRIF had normal bronchoconstriction response to aerogenic endotoxin (Figure 1c). Therefore, both adaptor proteins TIRAP and MyD88 play a critical and nonredundant role in the TLR4-dependent response for acute bronchoconstriction induced by intranasal instillation of LPS, while TRIF signaling is not required.

\section{Neutrophil Recruitment into the Lung upon Endotoxin Challenge Depends on TIRAP and MyD88, but not TRIF Signaling}

We then asked whether the absence of bronchoconstriction in TIRAP $-/-$ mice is associated with a reduced neutrophil recruitment into the lung. No neutrophils were detected in the BAL fluid of
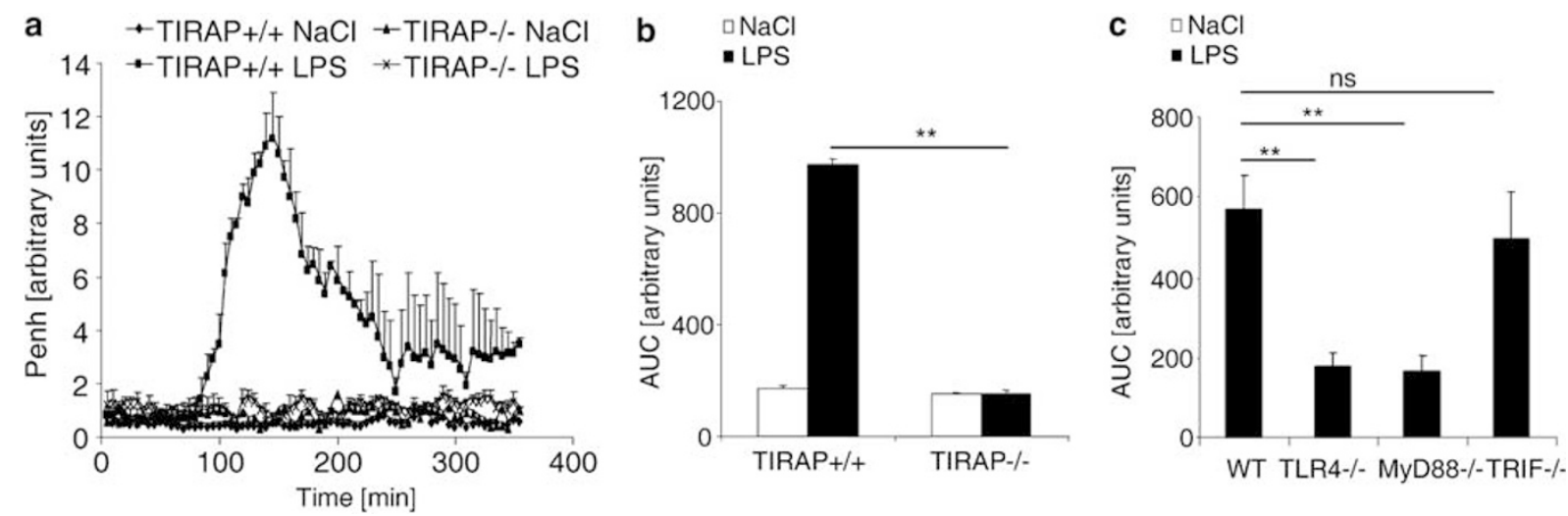

Figure 1 Absence of LPS-induced bronchoconstriction in mice deficient for TIRAP or MyD88, not TRIF. (a) Bronchoconstriction in wildtype control mice, but not in TIRAP-/- mice in response to aerogenic endotoxin ( $10 \mu \mathrm{g}$ LPS given by the i.n. route). The airways response is expressed as mean \pm s.e.m. of PenH $(P<0.01$ from 90 to $360 \mathrm{~min})$. (b) Area under the curve (AUC) measured from 60 to 180 min after endotoxin in TIRAP-/- and wild-type mice. (c) Absence of bronchoconstriction (AUC) in MyD88 and TLR4-/- mice, but not in TRIF - / - mice. Data are from one experiment representative of two independent experiments $\left(n=8\right.$ mice per group; ${ }^{*} P<0.01$; ns, not significant). 
TIRAP-/ - mice and MyD88-/- mice at $24 \mathrm{~h}$ upon intranasal endotoxin administration, in contrast to wild-type controls which had high neutrophil counts in the BAL (Figure 2a). In order to quantify neutrophil recruitment in the lung, myeloperoxidase (MPO) activity was determined in lung tissue homogenates. While wild-type mice showed a significant MPO activity $24 \mathrm{~h}$ after LPS challenge, TIRAP-/ - and MyD88-/- mice had essentially no MPO activity in lungs (Figure 2b), similar to untreated controls. The recruitment of neutrophils was not merely delayed in TIRAP-/ - mice as MPO activity did not increase at $24 \mathrm{~h}$ and $72 \mathrm{~h}$ (data not shown). Finally, endothelial damage was assessed by the measurement of protein leak into the BAL fluid. Protein levels were increased in wild-type mice, but not in TIRAP-/ - and in MyD88-/ - mice (Figure 2c) $24 \mathrm{~h}$ after endotoxin administration.

To assess the contribution of the MyD88-independent signaling, we tested TRIF deficient mice in the same conditions. Trif ${ }^{\text {Lps } 2}$ homozygote mutant mice had essentially normal neutrophil recruitment, lung MPO activity and protein levels in the BAL fluid in response to aerogenic endotoxin $3 \mathrm{~h}$ after endotoxin administration (Figure 2d-f). Therefore, TIRAPMyD88 signaling is critical for LPS-induced acute neutrophil sequestration into the lung parenchyma and the bronchoalveolar space, and for the microvascular damage resulting in protein leak, while TRIF signaling is dispensable.

\section{TIRAP and MyD88 are Essential for TNF, IL-12 p40 and KC Secretion in the Airways}

MyD88 is critical to signal some LPS-induced Th1 cytokine responses in murine BMDM in vitro. ${ }^{38} \mathrm{We}$ show that the production of TNF, IL-12p40 and NO by LPS activated BMDM is also TIRAP-dependent, as TIRAP-/ - macrophages are unresponsive to LPS in vitro (Figure 3a). We then quantified the production of cytokines and chemokines in BAL fluid and lung homogenates after intranasal endotoxin administration. Increased levels of TNF and $\mathrm{KC}$ at $3 \mathrm{~h}$ and IL-12 p40 at $24 \mathrm{~h}$ after endotoxin challenge were found in the BAL fluid of wild-type mice which were significantly reduced in TIRAP $-/-$ mice (Figure 3b), reminiscent of the results recently reported in MyD88-/- mice. ${ }^{28}$ Pulmonary TNF, IL12 p40 and KC production in response to local endotoxin was also severely impaired in TIRAP-/mice (Figure 3c). Therefore, both TIRAP and MyD88
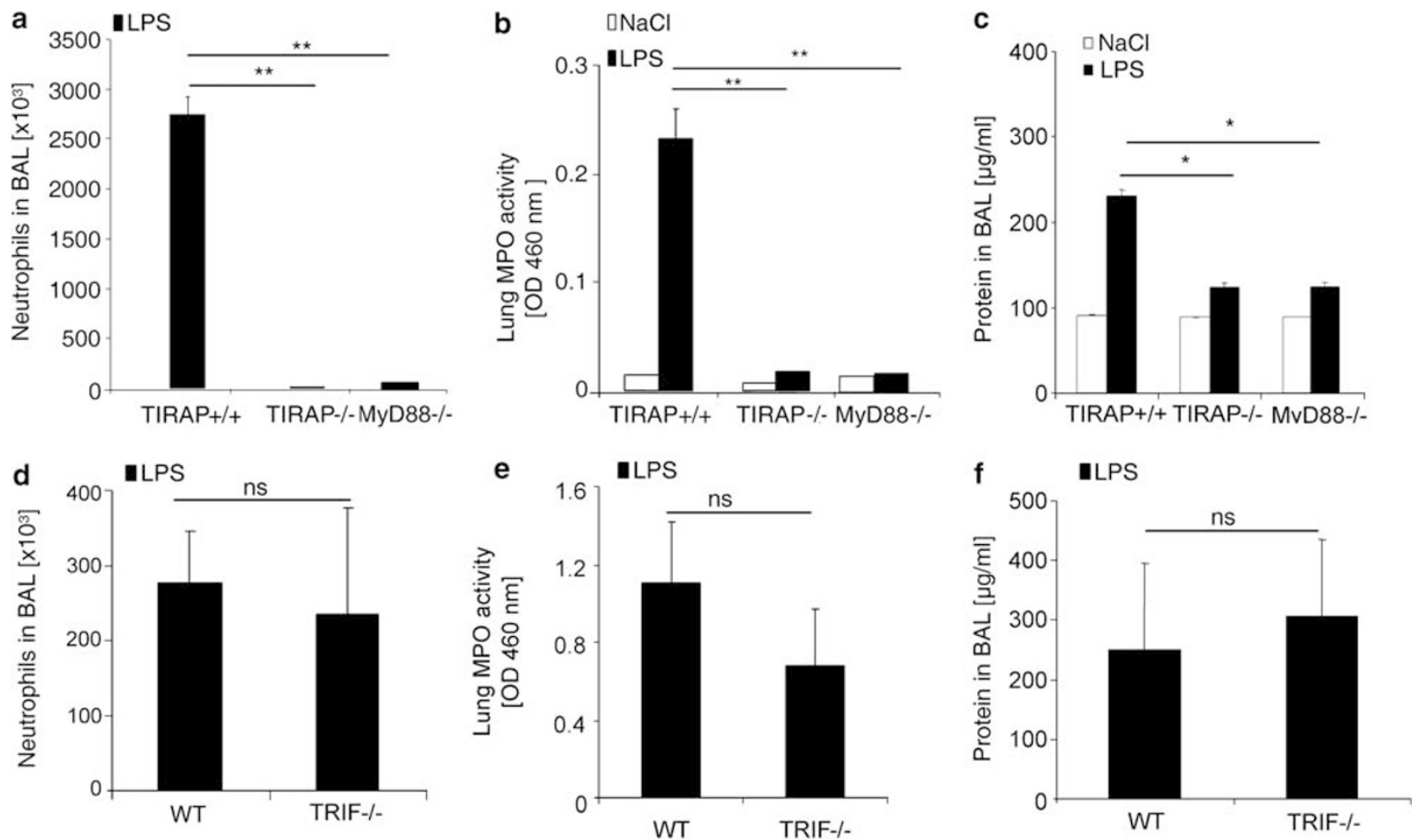

Figure 2 Reduced neutrophil recruitment and vascular leakage in the lung of LPS-challenged TIRAP-/- and MyD88-/- mice. (a) Neutrophil counts in the BALF were assessed $24 \mathrm{~h}$ after LPS administration ( $10 \mu \mathrm{g}$, i.n.) in wild-type (TIRAP $+/+$ ), TIRAP-/ - and MyD88-/ - mice. (b) Pulmonary MPO activity was measured in order to quantify the neutrophils in the lung tissue $24 \mathrm{~h}$ after endotoxin challenge. (c) Total protein levels were measured in the BALF $24 \mathrm{~h}$ after endotoxin challenge. (d) Neutrophil counts in the BALF were assessed $3 \mathrm{~h}$ after LPS administration (10 $\mu \mathrm{g}$, i.n.) in TRIF deficient mice. (e) Pulmonary MPO activity was measured $3 \mathrm{~h}$ after endotoxin challenge in lung of Trif ${ }^{L p s}$ homozygote mutant mice. (f) Total protein levels were measured in the BALF $3 \mathrm{~h}$ after endotoxin challenge. Data are representative of three independent experiments and are expressed as mean values \pm s.d. $\left(n=4\right.$ mice per group; ${ }^{*} P<0.05$; ${ }^{*} P<0.01$; nd, not detected; ns, not significant). 

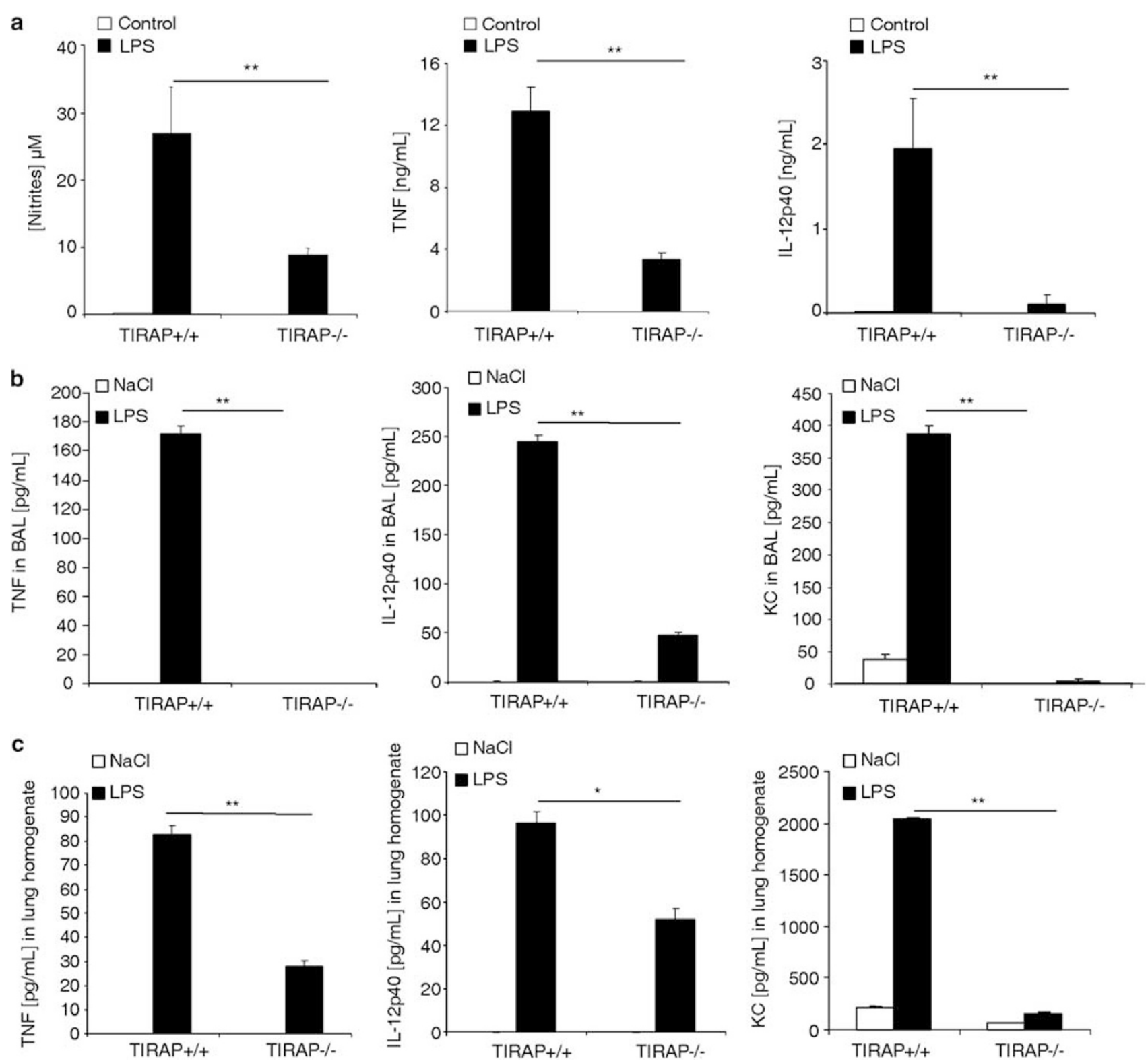

Figure 3 TIRAP-dependent airway TNF, IL-12p40 and KC production upon endotoxin challenge. (a) BMDM from TIRAP deficient and control mice were stimulated with endotoxin $(100 \mathrm{ng} / \mathrm{ml})$, or medium alone (control) and the supernatants assessed for TNF, IL-12p40 and KC production at $24 \mathrm{~h}$ by ELISA. (b) TNF, IL-12p40 and KC levels were determined by ELISA in the BAL fluid and (c) in lung homogenate from TIRAP-/ - and wild-type mice after LPS ( $10 \mu \mathrm{g}$, i.n.; black bars) or saline (open bars) administration. The data represent mean values \pm s.d. from three independent experiments $\left(n=4\right.$ mice per group; $\left.{ }^{*} P<0.05 ;{ }^{*} P<0.01\right)$.

adaptors are essential for the synthesis of TNF, IL-12 p40 and of the chemokine KC upon aerogenic endotoxin administration.

\section{Endotoxin-Induced Neutrophil Recruitment and Lung Injury is TIRAP and MyD88 Dependent}

Finally, we asked whether the neutrophil recruitment in the capillary and alveolar space and the damage of lung architecture seen upon endotoxin administration is TIRAP-dependent. The micrographs of the lung $24 \mathrm{~h}$ after endotoxin revealed a complete absence of neutrophils in the capillaries and alveolar space in TIRAP-/ - mice (Figure 4a), similar to MyD88-/- mice (Noulin et $a{ }^{28}$ and data not shown). The lung damage reflected by epithelial cell activation and focal necrosis, disruption of alveolar septae and focal edema was found in wildtype mice, but absent in TIRAP-/- (Figure 4a) and MyD88-/- mice (data not shown). Neutrophil infiltration and alveolar damage were quantified on microscopic sections. Lungs from TIRAP $-/-$ and MyD88-/- mice exhibited neither disruption of alveolar septae nor neutrophil in alveoli, while wild-type mice showed prominent neutrophil 
recruitment into the alveolar space, epithelial cell activation and disruption of alveolar septae after endotoxin administration (Figure $4 \mathrm{~b}-\mathrm{d}$ ).

Therefore, both TIRAP and MyD88 are pivotal to endotoxin-induced lung architecture damage and neutrophil recruitment.

\section{Endotoxin-Induced Airway Response is Independent-of IL-1 and IL-18 Receptor Signaling}

MyD88 is not only a common adaptor of most TLRs beside TLR3, but it is also involved in the signaling of IL-1 and IL-18 receptors. The extreme sensitivity of MyD88 deficient mice in several infectious models turned out to be due to the absence of functional IL-1R/IL-18R signaling, shedding new light on the prominent role of IL-1 in some innate responses (Gamero and Oppenheim, 2006; our unpublished observations). Here, the similar effects observed in TIRAP and MyD88 deficient mice upon intranasal endotoxin challenge strongly suggest that the role of MyD88 in this condition is linked to its contribution to TLR4 signaling pathway. To address this point and rule out a contribution of MyD88 through IL-1 or IL-18 receptor signaling, we a
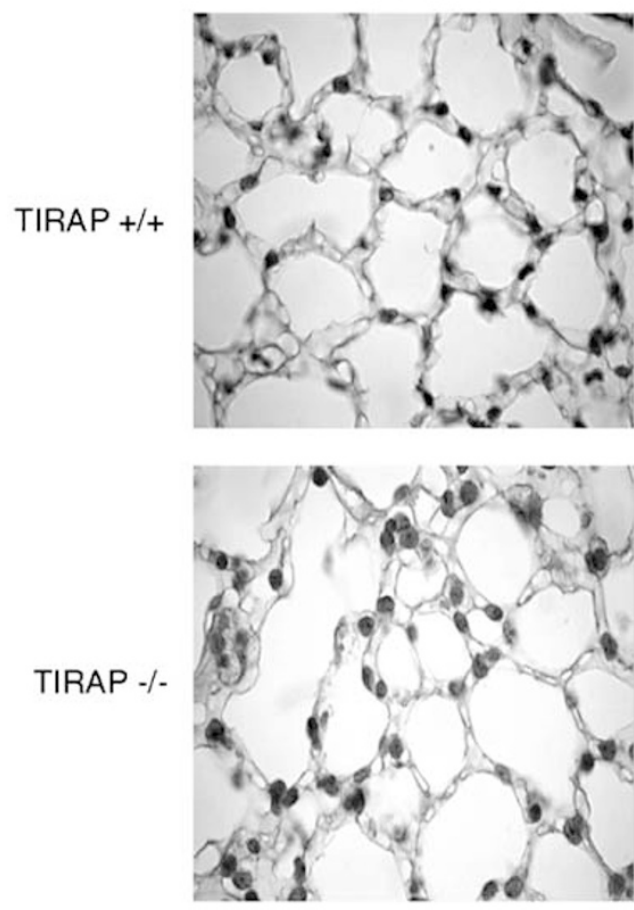

$\times 100$
LPS
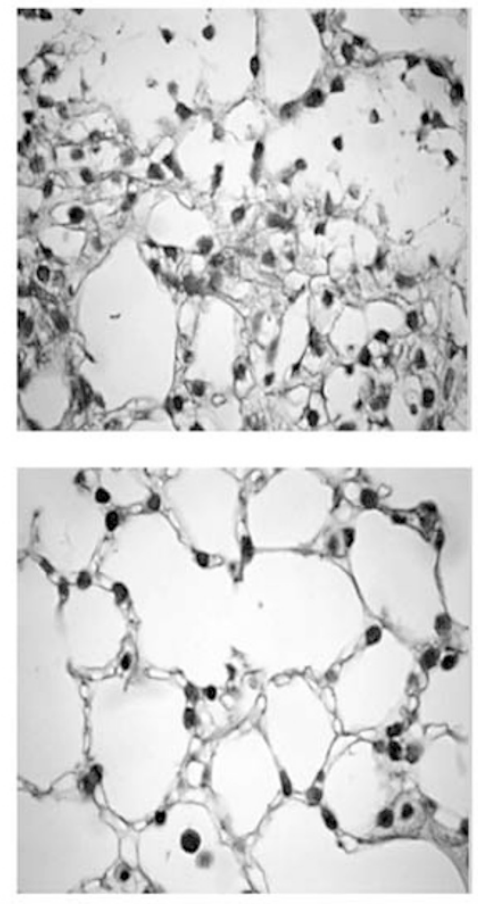

$X 100$ b Disruption of alveolar septae

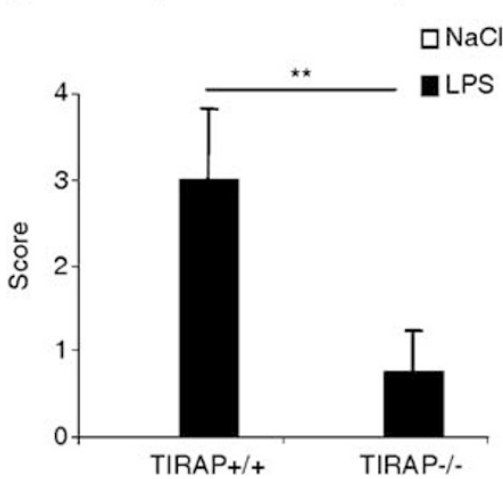

C

C Activation of alveolar epithelium

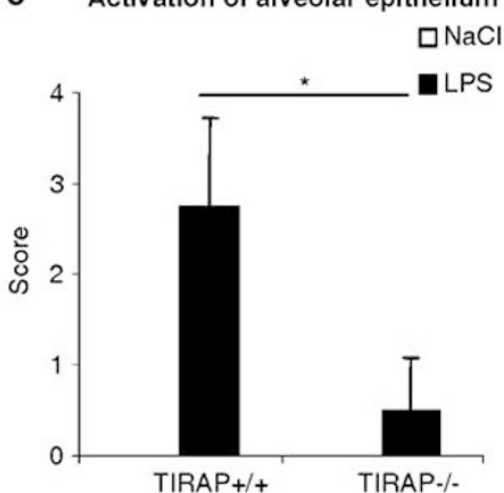

d Neutrophils in alveoli $\square \mathrm{NaCl}$

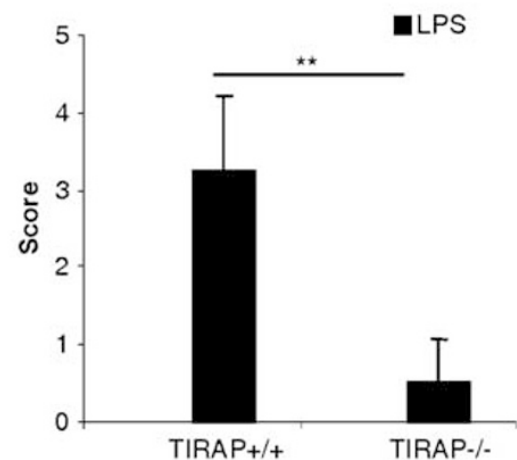

Figure 4 TIRAP- and MyD88-dependent inflammation and lung injury. The lungs of TIRAP-/ - mice were analyzed by microscopy $24 \mathrm{~h}$ after saline or LPS administration (10 $\mu$ g, i.n.). (a) Neutrophil infiltration, disruption of microarchitecture and activation alveolar epithelia observed in wild-type mice, were absent in TIRAP-/ - mice. Representative lung sections are shown at magnification $\times 100$. The lesions induced by endotoxins were assessed semiquantitatively (see Materials and methods): disruption of microarchitecture (b), activation of alveolar epithelial cells (c), neutrophils infiltration, (d) were essentially absent in TIRAP-/- mice. Results are expressed as mean lesion score \pm s.d. $\left(n \geq 5\right.$ mice per group; $\left.{ }^{*} P<0.05 ;{ }^{*} P<0.01\right)$. 
investigated the response of IL-1R1 and IL-18R deficient mice to aerogenic endotoxin. Unlike MyD88-/- mice, IL-1R1-/- mice and IL-18R-/mice responded to intranasal endotoxin with bronchoconstriction and an intense neutrophil recruitment to the lung and the bronchoalveolar space (Figure 5a-c). Moreover, bronchoconstriction and neutrophil recruitment were also observed in caspase-1 (ICE) deficient mice, ${ }^{34}$ which do not convert their precursors into active IL-1 $\beta$ and IL-18 (Figure 5a-c). These data demonstrate that IL-1 and IL-18 receptors are dispensable for bronchoconstriction and neutrophil recruitment into the airways upon aerogenic LPS exposure. Therefore, TIRAP together with MyD88 is critical for TLR4 signaling resulting in acute pulmonary inflammatory response to local endotoxin, but neither IL-1 $\beta$ nor IL-18 receptor signaling is essential for this response.

\section{Discussion}

It has been proposed that distinct combinations of adaptor molecules involved in the signaling platforms differ among TLRs and contribute to provide the observed specificity of the response induced by different TLR agonists. ${ }^{39,40}$ Endotoxin signals an inflammatory response by ligation to the CD14-TLR4 receptor complex recruiting several adaptor molecules and activating several kinases resulting in cell activation. TLR4 activation by endotoxin engages MyD88, TIRAP, TRIF and TRAM adaptor molecules, which induce differential activation programs in the cell. ${ }^{41}$ Here, we show that both TIRAP and MyD88 adaptor molecules are essential and nonredundant adaptors of TLR4 for endotoxin induced lung bronchoconstriction and acute airway inflammation while TRIF and the MyD88-dependent IL-1R and IL-18R pathways are dispensable for this response.

We recently demonstrated that MyD88 signaling is critical for endotoxin induced airway inflammation and that both MyD88 expressed in hemopoietic and resident cells are necessary for this response. ${ }^{28}$ Several studies have shown that TIRAP is a critical component of the TLR4 signaling cascade to LPS in isolated cells ${ }^{19}$ but less is known about the role of TIRAP in lung immune response against LPS. A recent report documented the implication of TIRAP in the lung inflammatory response to LPS or live E. coli but no information was given on the LPSinduced bronchoconstrictive response. ${ }^{31}$ Further we documented some mechanistic differences in the induction of bronchoconstriction vs inflammatory cell recruitment upon LPS administration. ${ }^{9}$ Indeed, in TNF deficient mice bronchoconstriction was abrogated, but not the neutrophil sequestration in the lung. Thus LPS provokes acute bronchoconstriction which is TNF-dependent and p38 MAPKmediated, while the neutrophil recruitment is independent of TNF although it also depends on LPS/TLR4-induced signals mediated by p38 MAPK.
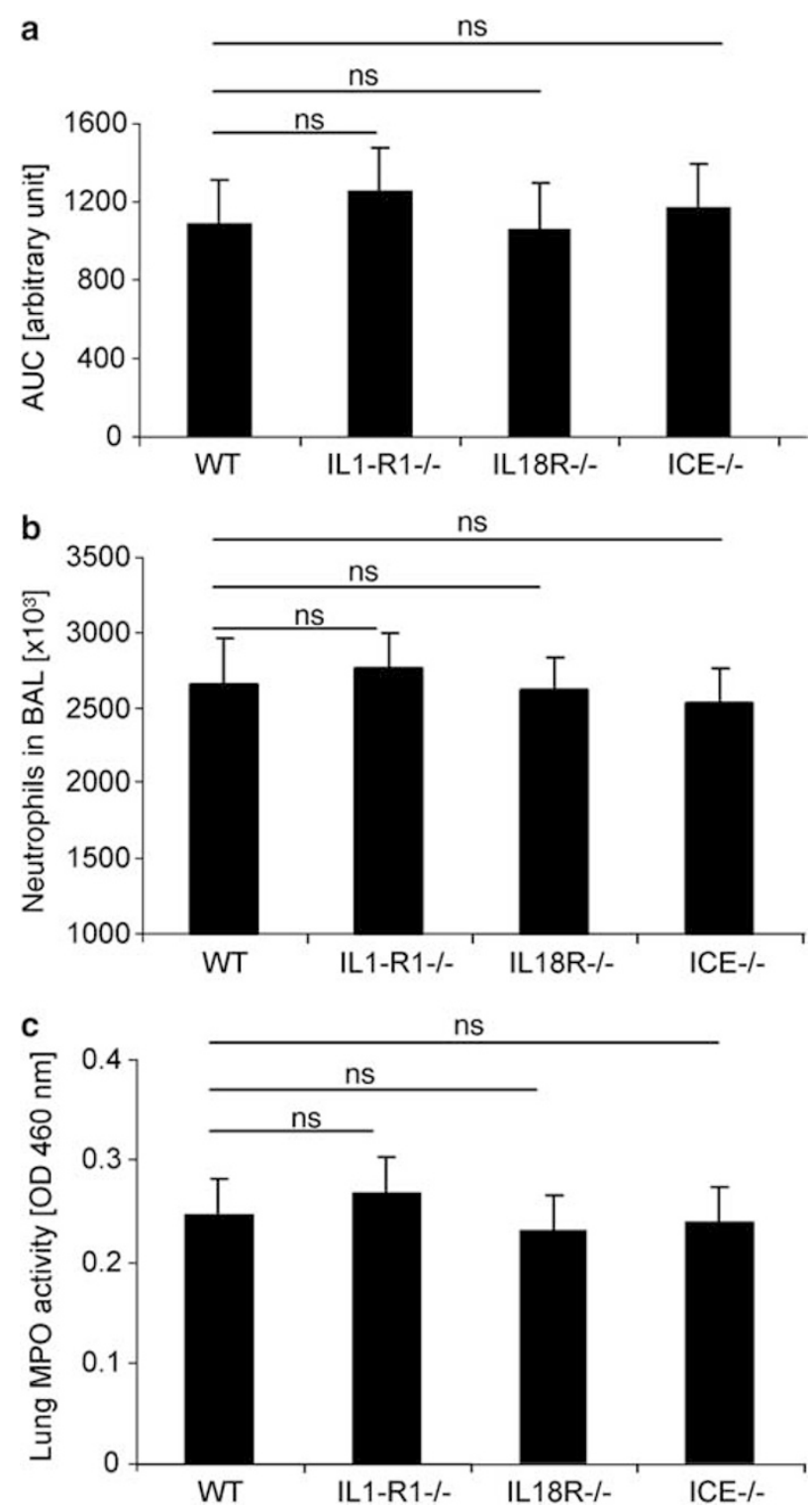

Figure 5 Bronchoconstriction and neutrophil recruitment in the absence of IL-1R1, IL-18R or caspase 1. IL-1R1-/-, IL-18R-/-, caspase $1-/-$ and wild-type mice received endotoxin at $10 \mu \mathrm{g}$ by the i.n. route, and bronchoconstriction was analyzed by $\mathrm{PenH}$ levels for $3 \mathrm{~h}(\mathbf{a})$. The airways response expressed as mean \pm s.e.m. of AUC was normal in IL-1R1-/-, IL-18R1-/-, caspase-1 (ICE)-/and wild-type mice $(P<0.05$ from 110 to $360 \mathrm{~min})$. The airway response was significantly higher in IL-1R- - mice $(P<0.05$ from 90 to $360 \mathrm{~min}$ ). (b) Neutrophil recruitment was assessed at $24 \mathrm{~h}$ in BALF and (c) in lung by MPO activity in lung homogenates. Data are representative of three independent experiments and are expressed as mean values \pm s.d. $\left(n=4\right.$ mice per group; $\left.{ }^{*} P<0.05\right)$. No significant difference in neutrophil recruitment was noted between the groups.

Here, we explored whether the adaptor TIRAP is required for the different aspects of TLR4-dependent airway response to endotoxin and investigated a possible contribution by TRIF signaling. We demonstrated that TIRAP is absolutely necessary to the LPS-induced MyD88-dependent inflammatory responses into the lung in mice and that MyD88 cannot 
compensate the lack of TIRAP. We showed that both adaptor proteins TIRAP and MyD88 play a critical and nonredundant role in the TLR4-dependent response for acute bronchoconstriction, for neutrophil sequestration into the lung parenchyma and the bronchoalveolar space, for the microvascular damage resulting in protein leak and for the synthesis of TNF, IL-12 p40 and chemokine KC, upon aerogenic endotoxin, while TRIF signaling is not required. The role of TIRAP on direct LPS-induced bronchoconstriction was not investigated before. Therefore, TLR4 signaling pathway requires both MyD88 and TIRAP adaptors, which are non-redundant, for an in vivo endotoxin response leading to severe airway injury.

MyD88 is not only a common adaptor of TLRs, but it is also involved in the signaling of IL-1 and IL-18 receptors. The fact that similar effects were observed in mice deficient for either TIRAP or MyD88 upon intranasal endotoxin challenge strongly supported the notion that the implication of MyD88 in this response was due to its contribution to TLR4 signaling. To further establish this point we ruled out a contribution of MyD88 through either IL-1 or IL-18 signaling. Indeed, mice deficient for IL-1R1, IL-18R or caspase- 1 that lack active IL-1 $\beta$ and IL-18 maturation, had a normal response to aerogenic endotoxin, with bronchoconstriction and intense neutrophil recruitment to the lung parenchyma and bronchoalveolar space, much in contrast to the abrogated response seen in MyD88 or TIRAP deficient mice. Our data clearly established that IL-1 and IL-18 receptors are dispensable for the airway response. Therefore, MyD88 is involved, together with TIRAP, in TLR4 signaling resulting in acute pulmonary inflammatory response to local endotoxin, but the MyD88-dependent, IL-1 and IL-18 receptor signaling is dispensable for this response.

Molecular modeling has suggested that TIRAP, MyD88 and TLR4 receptor may form a heterotetrameric complex, where TLR4 homodimerises and which, together with accessory molecules such as LBP, MD2 and CD14, allowing LPS recognition and downstream signaling. The role of TIRAP in TLR4 signaling was believed to be structural, acting as a linker of MyD88 to TLR4, similar to TRAM that may act by bridging TRIF and TLR4. Both TIRAP and TRAM have been found to associate constitutively with TLR $4^{22,42}$ and may serve as platform-forming components responsible for recruitment of the larger adapters MyD88 and TRIF, respectively, that in turn, recruit downstream effector molecules such as IRAK-1 and IRAK-4 to MyD88 and TBK-1, TRAF6 and IKK-e to TRIF via non-TIR domains. Nevertheless, TIRAP and MyD88 bind to different sites on TLR 2 and 4, a finding consistent with a cooperative role of the two adaptors in signaling. Moreover, a novel feature in TIRAP that distinguishes it from MyD88 was identified which implicates TIRAP in downstream signaling through the engagement of distinct kinases. Indeed, putative TRAF6 interaction sites were identified in TIRAP but not in MyD88 suggesting a specific role of TIRAP in TLR2- and TLR4-mediated regulation of $\mathrm{NF}-\kappa \mathrm{B}$-dependent gene transcription via interaction of its TIR domain with TRAF6. ${ }^{43}$ Interaction between TIRAP and TRAF6 is necessary for p65-mediated transactivation of $\mathrm{NF}-\kappa \mathrm{B}$, to control gene expression which is of critical importance to regulate the proinflammatory response. Transactivation by p65 is dependent of Bruton's tyrosine kinase (Btk), a critical tyrosine kinase in LPS signaling which has been shown to interact with, and phosphorylates TIRAP and TRAM. ${ }^{44-48}$ TIRAP was also recently implicated in negative regulation of TLR4 signaling. Membranebound form of ST2, a member of the TIR family that does not activate NK-kB, was shown to negatively regulate TLR4-mediated NF- $\kappa \mathrm{B}$ activation by sequestrating the adaptors TIRAP and MyD88, implicating TIRAP as a target for inhibition of TLR-4 signaling. ${ }^{49}$ In the context of these new functions of TIRAP beyond the sole adaptor function, our study shows that TIRAP is an essential component for the LPSinduced MyD88-dependent inflammatory lung responses in mice and that MyD88 cannot compensate the lack of TIRAP.

Recent evidence suggests that TLR4 activation results in phophorylation of TIRAP/Mal ${ }^{44}$ and TRAM. ${ }^{45}$ Further, LPS activation induced TIRAP/ Mal localization to the plasma membrane by binding to PIP2 (phophatidylinositol 4,5-biphosphate) sites. This binding recruits MyD88 to TLR4, which is the first evidence of a cross-talk between TLR signaling and phopholipid metabolism. ${ }^{50}$ Further, for the MyD88-independent pathway of TLR4 activation recent evidence suggests that TRAM containing a N-terminal myristyolation site is targeted to plasma membrane colocalized to TLR4. ${ }^{51}$ Upon activation membrane bound TRAM recruits TRIF to TLR4 and activates interferon regulatory factor 3 (IRF-3) and induced type 1 interferons and delayed $\mathrm{NF}-\kappa \mathrm{B}$ activation. $^{52}$ Therefore, at least for TLR4 signaling the role of the key adaptor proteins TIRAP/Mal and TRAM is getting clearer and completes the most recent reviews. ${ }^{16,17}$

Although LPS induces a MyD88-independent response, which is TRIF-mediated, and result in type I interferon production in vitro, ${ }^{41}$ our data exclude a major role for TRIF-dependent, MyD88and TIRAP-independent signaling mechanisms in the inflammatory lung response to endotoxin. Therefore, endotoxin-induced bronchoconstriction, injury of the alveolar epithelium and endothelium that result in neutrophil recruitment into the lung and the bronchoalveolar space, and vascular leak, are strictly dependent on the presence of both adaptor proteins, MyD88 and TIRAP but are independent of the type I interferon inducing, TRIF adaptor pathway.

In conclusion, our data demonstrate that both TIRAP and MyD88 adaptor molecules play a critical 
and non-redundant role in bronchoconstriction, proinflammatory cytokine secretion, vascular leak and neutrophil recruitment in response to aerogenic LPS while TRIF-dependent signaling is dispensable for the endotoxin-induced airway response.

\section{Acknowledgements}

Grant support by the French 'Ministère de l'Education Nationale, de la Recherche et de la Technologie' (to Nicolas Noulin), Fondation de la Recherche and CNRS. The work was also supported by grants from Le Studium (Orleans), and Fondation de la Recherche Médicale. We acknowledge the skilled technical assistance of Marielle Maret and Lizette Fick.

\section{Disclosures}

The authors have no financial conflict of interest.

\section{References}

1 Schwartz DA, Thorne PS, Yagla SJ, et al. The role of endotoxin in grain dust-induced lung disease. Am J Respir Crit Care Med 1995;152:603-608.

2 Kennedy SM, Christiani DC, Eisen EA, et al. Cotton dust and endotoxin exposure-response relationships in cotton textile workers. Am Rev Respir Dis 1987; 135:194-200.

3 Donham K, Haglind P, Peterson Y, et al. Environmental and health studies of farm workers in Swedish swine confinement buildings. Br J Ind Med 1989;46:31-37.

4 Michel O, Kips J, Duchateau J, et al. Severity of asthma is related to endotoxin in house dust. Am J Respir Crit Care Med 1996;154:1641-1646.

5 Liu AH. Something old, something new: indoor endotoxin, allergens and asthma. Paediatr Respir Rev 2004;5(Suppl A):S65-S71.

6 Kline JN, Cowden JD, Hunninghake GW, et al. Variable airway responsiveness to inhaled lipopolysaccharide. Am J Respir Crit Care Med 1999;160:297-303.

7 Arbour NC, Lorenz E, Schutte BC, et al. TLR4 mutations are associated with endotoxin hyporesponsiveness in humans. Nat Genet 2000;25:187-191.

8 Lefort J, Motreff L, Vargaftig BB. Airway administration of Escherichia coli endotoxin to mice induces glucocorticosteroid-resistant bronchoconstriction and vasopermeation. Am J Respir Cell Mol Biol 2001;24: 345-351.

9 Schnyder-Candrian S, Quesniaux VF, Di Padova F, et al. Dual effects of p38 MAPK on TNF-dependent bronchoconstriction and TNF-independent neutrophil recruitment in lipopolysaccharide-induced acute respiratory distress syndrome. J Immunol 2005;175: 262-269.

10 Andonegui G, Goyert SM, Kubes P. Lipopolysaccharide-induced leukocyte-endothelial cell interactions: a role for CD14 vs toll-like receptor 4 within microvessels. J Immunol 2002;169:2111-2119.
11 Andonegui G, Bonder CS, Green F, et al. Endotheliumderived Toll-like receptor-4 is the key molecule in LPS-induced neutrophil sequestration into lungs. J Clin Invest 2003;112:1264.

12 Lefort J, Singer M, Leduc D, et al. Systemic administration of endotoxin induces bronchopulmonary hyperreactivity dissociated from TNF-alpha formation and neutrophil sequestration into the murine lungs. J Immunol 1998;161:474-480.

13 Togbe D, Schnyder S, Schnyder B, et al. TLR4 gene dosage contributes to endotoxin induced acute respiratory inflammation. J Leukoc Biol 2006;80: (in press).

14 Yamamoto M, Sato S, Mori K, et al. Cutting edge: a novel Toll/IL-1 receptor domain-containing adapter that preferentially activates the IFN-beta promoter in the Toll-like receptor signaling. J Immunol 2002;169: 6668-6672.

15 Hoebe K, Du X, Georgel P, et al. Identification of Lps2 as a key transducer of MyD88-independent TIR signalling. Nature 2003;424:743-748.

16 Beutler B, Jiang Z, Georgel P, et al. Genetic analysis of host resistance: toll-like receptor signaling and immunity at large. Annu Rev Immunol 2006;24: 353-389.

17 Akira S, Uematsu S, Takeuchi O. Pathogen recognition and innate immunity. Cell 2006;124:783-801.

18 Kawai T, Adachi O, Ogawa T, et al. Unresponsiveness of MyD88-deficient mice to endotoxin. Immunity 1999;11:115-122.

19 Yamamoto M, Sato S, Hemmi H, et al. Essential role for TIRAP in activation of the signalling cascade shared by TLR2 and TLR4. Nature 2002;420:324-329.

20 Oshiumi H, Sasai M, Shida K, et al. TIR-containing adapter molecule (TICAM)-2, a bridging adapter recruiting to toll-like receptor 4 TICAM-1 that induces interferon-beta. J Biol Chem 2003;278:49751-49762.

21 Horng T, Barton GM, Flavell RA, et al. The adaptor molecule TIRAP provides signalling specificity for Toll-like receptors. Nature 2002;420:329-333.

22 Fitzgerald KA, Palsson-McDermott EM, Bowie AG, et al. Mal (MyD88-adapter-like) is required for Toll-like receptor-4 signal transduction. Nature 2001;413:78-83.

23 Horng T, Barton GM, Medzhitov R. TIRAP: an adapter molecule in the Toll signaling pathway. Nat Immunol 2001;2:835-841.

24 Yamamoto $M$, Sato S, Hemmi $H$, et al. TRAM is specifically involved in the Toll-like receptor 4mediated MyD88-independent signaling pathway. Nat Immunol 2003;4:1144-1150.

25 Yamamoto M, Sato S, Hemmi H, et al. Role of adaptor TRIF in the MyD88-independent toll-like receptor signaling pathway. Science 2003;301:640-643.

26 Guillot L, Medjane S, Le-Barillec K, et al. Response of human pulmonary epithelial cells to lipopolysaccharide involves Toll-like receptor 4 (TLR4)-dependent signaling pathways: evidence for an intracellular compartmentalization of TLR4. J Biol Chem 2004;279: 2712-2718 (Epub 2003 Nov 2714).

27 Piggott DA, Eisenbarth SC, Xu L, et al. MyD88dependent induction of allergic Th2 responses to intranasal antigen. J Clin Invest 2005;115:459-467.

28 Noulin N, Quesniaux VF, Schnyder-Candrian S, et al. Both hemopoietic and resident cells are required for MyD88-dependent pulmonary inflammatory response to inhaled endotoxin. J Immunol 2005;175: 6861-6869. 
29 Zeisel MB, Druet VA, Sibilia J, et al. Cross talk between MyD88 and focal adhesion kinase pathways. J Immunol 2005;174:7393-7397.

30 Gamero AM, Oppenheim JJ. IL-1 can act as number one. Immunity 2006;24:16-17.

31 Jeyaseelan S, Manzer R, Young SK, et al. Toll-IL-1 receptor domain-containing adaptor protein is critical for early lung immune responses against Escherichia coli lipopolysaccharide and viable Escherichia coli. J Immunol 2005;175:7484-7495.

32 Glaccum MB, Stocking KL, Charrier K, et al. Phenotypic and functional characterization of mice that lack the type I receptor for IL-1. J Immunol 1997;159:33643371 .

33 Hoshino K, Tsutsui H, Kawai T, et al. Cutting edge: generation of IL-18 receptor-deficient mice: evidence for IL-1 receptor-related protein as an essential IL-18 binding receptor. J Immunol 1999;162:5041-5044.

34 Kuida K, Lippke JA, Ku G, et al. Altered cytokine export and apoptosis in mice deficient in interleukin-1 beta converting enzyme. Science 1995;267:2000-2003.

35 Hoshino K, Takeuchi O, Kawai T, et al. Cutting edge: Toll-like receptor 4 (TLR4)-deficient mice are hyporesponsive to lipopolysaccharide: evidence for TLR4 as the Lps gene product. J Immunol 1999;162:3749-3752.

36 Couillin I, Maillet I, Vargaftig BB, et al. Arthropodderived histamine-binding protein prevents murine allergic asthma. J Immunol 2004;173:3281-3286.

37 Muller M, Eugster HP, Le Hir M, et al. Correction or transfer of immunodeficiency due to TNF-LT alpha deletion by bone marrow transplantation. Mol Med 1996;2:247-255.

38 Nicolle DM, Pichon X, Bouchot A, et al. Chronic pneumonia despite adaptive immune response to Mycobacterium bovis BCG in MyD88-deficient mice. Lab Invest 2004;84:1305-1321.

39 Vogel SN, Fitzgerald KA, Fenton MJ. TLRs: differential adapter utilization by toll-like receptors mediates TLR-specific patterns of gene expression. Mol Interv 2003;3:466-477.

40 Akira S, Takeda K. Toll-like receptor signalling. Nat Rev Immunol 2004;4:499-511.

41 Hirotani T, Yamamoto M, Kumagai Y, et al. Regulation of lipopolysaccharide-inducible genes by MyD88 and Toll/IL-1 domain containing adaptor inducing
IFN-beta. Biochem Biophys Res Commun 2005;328: 383-392.

42 Fitzgerald KA, Rowe DC, Barnes BJ, et al. LPS-TLR4 signaling to IRF-3/7 and NF-kappaB involves the toll adapters TRAM and TRIF. J Exp Med 2003;198:10431055.

43 Mansell A, Brint E, Gould JA, et al. Mal interacts with tumor necrosis factor receptor-associated factor (TRAF)-6 to mediate NF-kappaB activation by toll-like receptor (TLR)-2 and TLR4. J Biol Chem 2004;279:37227-37230.

44 Gray P, Dunne A, Brikos C, et al. MyD88 adapter-like (Mal) is phosphorylated by Bruton's tyrosine kinase during TLR2 and TLR4 signal transduction. J Biol Chem 2006;281:10489-10495.

45 McGettrick AF, Brint EK, Palsson-McDermott EM, et al. Trif-related adapter molecule is phosphorylated by PKC\{varepsilon\} during Toll-like receptor 4 signaling. Proc Natl Acad Sci USA 2006;103: 9196-9201.

46 Doyle SL, Jefferies CA, O’Neill LA. Bruton's tyrosine kinase is involved in p65-mediated transactivation and phosphorylation of p65 on serine 536 during NFkappaB activation by lipopolysaccharide. J Biol Chem 2005;280:23496-23501.

47 Jefferies CA, O’Neill LA. Bruton's tyrosine kinase (Btk)-the critical tyrosine kinase in LPS signalling? Immunol Lett 2004;92:15-22.

48 Gray P, Dunne A, Brikos C, et al. MyD88 adapter-like (Mal) is phosphorylated by Bruton's tyrosine kinase during TLR2 and TLR4 signal transduction. J Biol Chem 2006;281:10489-10495.

49 Brint EK, Xu D, Liu H, et al. ST2 is an inhibitor of interleukin 1 receptor and Toll-like receptor 4 signaling and maintains endotoxin tolerance. Nat Immunol 2004;5:373-379.

50 Kagan JC, Medzhitov R. Phosphoinositide-mediated adaptor recruitment controls Toll-like receptor signaling. Cell 2006;125:943-955.

51 Rowe DC, McGettrick AF, Latz E, et al. The myristoylation of TRIF-related adaptor molecule is essential for Toll-like receptor 4 signal transduction. Proc Natl Acad Sci USA 2006;103:6299-6304.

52 Fitzgerald KA, Chen ZJ. Sorting out Toll signals. Cell 2006;125:834-836. 